\title{
Guest editorial: Special issue on algorithms, systems and applications in mobile social networks
}

\author{
Haojin $\mathrm{Zhu}^{1} \cdot$ Kuai $\mathrm{Xu}^{2} \cdot \mathrm{Xiang} \mathrm{Lu}^{3}$
}

Published online: 18 April 2016

(C) Springer Science+Business Media New York 2016

Social Networks are a massively successful phenomenon, used by billions of users to interact. A new trend has appeared that social networks are undergoing the transition from traditional web-based social networking to mobile social networking by exploiting ubiquitous access of mobile Internet and mobile computing/communications as well as cloud-based applications and services. Research in network science and its application to the Internet has highlighted the interplay between technological networks and social networks. This interplay suggests a paradigm shift in the design of wireless communication systems and networks to incorporate social network theory and applications into such design. However, there are still significant technological challenges in the development of algorithms, systems, and applications for mobile social networks, and the purpose of this special issue is to highlight recent advances in this field.

Haojin Zhu

zhu-hj@cs.sjtu.edu.cn

Kuai Xu

kuai.xu@asu.edu

Xiang Lu

luxiang@iie.ac.cn

1 Shanghai Jiao Tong University, 800 Dongchuan Rd, Minhang Shanghai 200240, China

2 Arizona State University, Tempe, AZ 85281, USA

3 Institute of Information Engineering, Chinese Academy of Sciences, 52 Sanlihe Rd., Xicheng District Beijing 100864, China
Ten papers have been accepted from theopen call or selected from the outstanding papers from WASA'2015, which requires a significant improvement in terms of technical contribution. Their brief summaries are listed below.

"Delivering Mobile Social Content with Selective Agent and Relay Nodes in Content Centric Networks" byZejun Xu, Zhou Su, Qichao Xu, Qifan Qi, Tingting Yang, Jintian Li, Dongfeng Fang, and Bo Han, designs the next generation mobile social networks with content centric architecture by presenting a novel scheme to deliver the mobile social content by the selective agent nodes and relay nodes.

"A Method for Defensing Against Multi-source Sybil Attacks in VANET" by Xia Feng, Chun-yan Li, De-xin Chen, and Jin Tang, proposes an event based reputation system, which is expected to detect Sybil attacks with fabricated identities and stolen identities in the vehicular based mobile social networks.

"An Adaptive Resource Allocation Model in AntiMoney Laundering System" by Xintao Hong, Hongbin Liang, Zengan Gao, Hongwei Li, presents a novel Adaptive Anti-Money Laundering (AML) Resource Allocation Model based on Semi-Markov Decision Process, which is expected to allocate AML resources optimally in AML resource allocation domain to analyze the suspicious transaction report sent from Financial Institutions in mobile social networks.

"Serial Number Based Encryption and Its Application for Mobile Networks" by Rong Ma, Zhenfu Cao, presents a lightweight functional public key encryption scheme and shows how to use it to enhance the security and privacy of applications in mobile networks. 
"Privacy-Preserving Design for Emergency Response Scheduling System in Medical Social Networks" by Wenbin Yu, Zhe Liu, Cailian Chen, Bo Yang, Xinping Guan, presents a privacy-preserving based scheduling scheme for emergency response system, which is expected to protect physiological data privacy, location privacy and personal attribute privacy in mobile social networks.

"Characterizing User Behaviors in Location-Based Find-and-Flirt Services: Anonymity and Demographics" by MinhuiXue, Limin Yang, Keith W. Ross, Haifeng Qian, takes an in-depth examination of the user anonymity and demographics in a popular mobile social networks, WeChat.

"An Ultra-Lightweight RFID Authentication Scheme for Mobile Commerce" by Kai Fan, Nan Ge, Yuanyuan Gong, Hui Li, Ruidan Su and Yintang Yang, presents an ultralightweight RFID authentication scheme, which only uses Bit and XOR operations to prevent the DDOS attack in mobile social networks.

"A Novel Approach for Inhibiting Misinformation Propagation in Human Mobile Opportunistic Networks" by Xiaoming Wang, Yaguang Lin, Yanxin Zhao, Lichen Zhang, JuhuaLiang, Zhipeng Cai, proposes a novel approach based on vaccination and treatment strategies for inhibiting misinformation propagation in mobile social networks.

"SOS: Real-time and Accurate Physical Assault Detection using Smartphone" by Zehao Sun, Shaojie Tang, He Huang, Zhenyu Zhu, Hansong Guo, Yu-e Sun, Liusheng Huang, investigates the issue of how to detect those severe and noninstantaneous physical assaults using accelerometer in smartphone by proposing a combinatorial classification scheme considering individuality of user's Activities of Daily Living and universality of differences between ADLs and assaults to most people.

"Finding Overlapping Communities based on Markov Chain and Link Clustering" by Xiaoheng Deng, Genghao $\mathrm{Li}$, and Mianxiong Dong, and Kaoru OT a proposes a novel MCLC algorithm to discover overlapping communities in mobile social networks.

Finally, we would like to thank all the authors who contributed manuscripts to our special issue, and all of the anonymous dedicated reviewers for their expert comments and time to help us make the final decisions. Without their valuable and strong support, we could not have made this special issue successful. We would like also to express our sincere gratitude to the PPNA EiC, Prof. Xuemin (Sherman) Shen, as well as Ms. Melissa Fearon, Ms. Ethel Dionela and Mr. Hector Nazario from the Springer Journal Editorial Office for helping us to publish this special issue to readers.

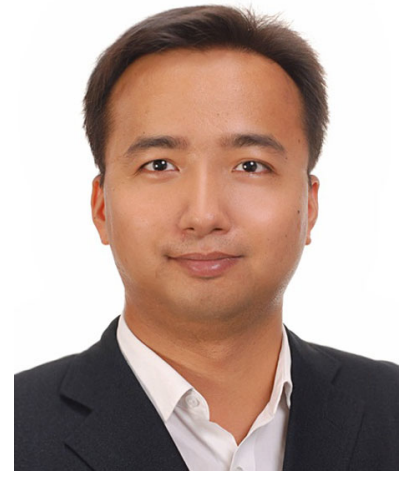

Haojin Zhu received his B.Sc. degree (2002) from Wuhan University (China), his M.Sc. (2005) degree from Shanghai Jiao Tong University (China), both in computer science and the $\mathrm{Ph}$.D. in Electrical and Computer Engineering from the University of Waterloo (Canada), in 2009. After that, he joined Shanghai Jiao Tong University as an Assistant Professor. He is currently an Associate Professor with Department of Computer Science and Engineering, Shanghai Jiao

Tong University, China. His current research interests include wireless network security and distributed system security. He published 31 international journal papers, including IEEE Trans. On Parallel and Distributed Systems, IEEE Trans. on Wireless Communication, IEEE Trans. on Vehicular Technology, IEEE Wireless Communications, IEEE Communications, and 55 international conference papers, including ACM MOBICOM, ACM MOBIHOC, IEEE INFOCOM, IEEE ICDCS, IEEE GLOBECOM, IEEE ICC, IEEE WCNC. He received the IEEE ComSoc Asia-Pacific Outstanding Young Researcher Award (2014), Top 100 Most Cited Chinese Papers Published in International Journals of 2014, Supervisor of Shanghai Excellent Master Thesis (2014), Distinguished Member of the IEEE INFOCOM 2015 Technical Program Committee, Outstanding Youth Post Expert Award for Shanghai Jiao Tong University, SMC-Young Research Award of Shanghai Jiao Tong University. He was a co-recipient of best paper awards of IEEE ICC 2007 and Chinacom 2008, and best paper nomination of Globecom'14. He serves as the Associate/Guest Editor of IEEE Internet of Things Journal, IEEE Wireless Communications, IEEE Network, Ad Hoc \& Sensor Wireless Networks, and Peer-to-Peer Networking and Applications. He is a senior member of IEEE and a member of ACM.

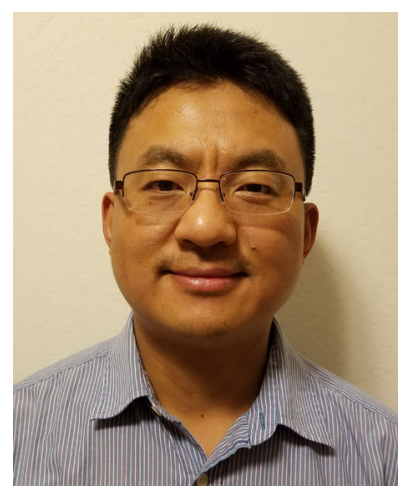

Kuai $\mathbf{X u}$ is an Associate Professor at the School of Mathematical and Natural Sciences, Arizona State University. He received his Ph.D. degree in computer science from the University of Minnesota in 2006, and his B.S. and M.S. degrees from Peking University, China, in 1998 and 2001. His research interests span the areas of Internet measurement, network security, cloud computing and online social networks. His research has resulted in over 50 papers in major conference and journals including ACM SIGCOMM and IEEE/ ACM Transactions on Networking, and three awarded United State patents. He served as the general co-chair of IEEE IPCCC 2013, the workshop co-chair of IEEE CCNC DCN 2011 and 2012, and the program cochair of IEEE IPCCC 2009, IEEE IPCCC 2010, and WASA 2015. He has also served on technical program committees for a number of major conferences including ACM Internet Measurement Conference, IEEE INFOCOM, IEEE GLOBECOM and IEEE ICCC. Dr. Xu is a senior member of IEEE and a member of ACM. 


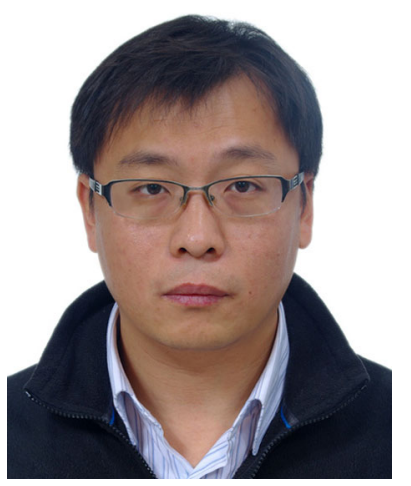

Xiang Lu is an Associate Professor at the Institute of Information Engineering, Chinese Academy of Sciences. He received his Ph.D. degree in computer science from Xidian University, China, in2013, and his B.S. degree in electrical engineering also from Xidian University in 2005. From 2009 to 2012, he was a visiting Ph.D. student in the Department of Electrical and Computer Engineering, North Carolina State University. His current research is related to computer and network security, with an emphasis on performance and vulnerability analysis of security schemes in practical applications and systems. He is also working on cyber-physical system security, especially in those emerging areas, like the smart grid, video surveillance systems, and so on. He published multiple papers in major conference and journals, including IEEE INFOCOM, Globecom, IEEE Transaction on Smart Grid. He is the reviewer of a number of conference and journals, including IEEE INFOCOM and IEEE Transactions on Mobile Computing. He is also served on the technical program committee for IEEE ICC 2015. Dr. Lu is a member of ACM and IEEE. 